\title{
Elastic Percolation of Composite Structures With Regular Tessellations of Microstructure
}

\author{
MH. Zarei ${ }^{\mathrm{a}}$, V. Fadaei Naeini ${ }^{\mathrm{a}}$, M. Baghani ${ }^{\mathrm{a}}$, J. Jamali $^{\mathrm{b}}$, M. Baniassadi ${ }^{\mathrm{a}, \mathrm{c}} *$ \\ ${ }^{a}$ School of Mechanical Engineering, College of Engineering, University of Tehran, Tehran, Iran \\ ${ }^{\mathrm{b}}$ Shoushtar Branch Azad University, Shoushtar, Iran \\ ${ }^{c}$ University of Strasbourg, ICube/CNRS, 2 Rue Boussingault, 67000 Strasbourg, France
}

\begin{abstract}
Enhancing the mechanical properties of materials by adding inclusions (particles) into microstructure is a favorite research area for material science researchers. In this paper, effects of different geometrical shapes of inclusions on overall elastic properties of a microstructure with regular tessellations were investigated. Finite element method (FEM) was used to analyze the stretching and shearing behavior of the microstructure including particles with different geometrical shapes. The results show that the dependency of tensile and shearing properties of the microstructure on volume fraction of particles can completely have different trends in some ranges of volume fractions. Besides, it was observed that each regular tessellation can have a different effect in enhancement of mechanical properties. Also the effects of mechanical percolation on the elastic properties of microstructures were investigated. The presented approach opens a systematic method for investigating the enhanced properties of microstructures with different shapes of tessellations.
\end{abstract}

Keywords:

Enhanced Mechanical Properties, Inclusion, Microstructure, Percolation, Lower Bound Limit

\section{Introduction}

Enhanced mechanical properties have been observed in polymer nanocomposites at uncharacteristically low volume fractions. These effects are thought to be due, in part, to the significant scale effect of the matrix-particle interface region in nanocomposites [1-3]. This interface region occurs as a result of a perturbation of the properties of the matrix material due to the presence of the included particles. Factors that may cause this perturbation are, e.g., the quality of bonding between phases of the material, confinement of the matrix, or interference in the mobility of the flexible chains of the polymer [4-6].

A number of researchers have attempted to include more mechanics in modeling mechanical percolation. Early work included the Generalized Effective Media model [7], which interpolated between a mean field model, at low volume fractions, and percolation theory, above the percolation threshold. This model has been used to predict both electrical and mechanical percolation [6, 8]. The series-parallel model included an intermediate parameter that described the volume fraction of material that was active in the transfer of forces [9]. A limitation of both of these models is that a previously identified value for the percolation threshold is required as input. In some cases the influence of an interface region, as well as the effects of clustering using the concentric cylinder micromechanics model, but not in the context of percolation thresholds [10]. A hybrid numerical analytic model was used in to investigate polymer nanocomposites with complex microstructural configurations; the model included the effects of an interface as a third, independent phase, i.e., not linked to particle placement [11]. The relative influence of the competing and compounding effects of the spatial position/distribution of the particles (microstructure) and of the composite constitution (micromechanics) are examined [12].

Effects of geometrical properties of carbon nanotubes (such as length and diameter) on the percolation limit of nanocomposites were investigated in previous studies $[13,14]$. In some cases, these effects on percolation threshold can be calculated based on 3D computational simulations [13]. Also, analysis of percolation threshold in a type of 
polymers filled with penetrable and impenetrable graphite nanoplatelets (GNPs) is carried out by 3D Monte Carlo simulation [14]. Comparison with other experimental results [15, 16] indicates that their assumptions give satisfactory results.

Some of studies have used experimental data to generate correlation functions. These functions can be used to evaluate some of thermal or mechanical properties of nanocomposites. Statistical two and three point correlation function of a polymer/ $\mathrm{ZrO}_{2}$ nanocomposite material was calculated using small-angle X-ray scattering data [17]. A continuum approach was employed to estimate the effective thermal conductivity of this type of polymer nanocomposite. Comparison of results extracted by this methodology demonstrates a good agreement with other simulated results. In some cases, reconstruction of nanocomposites was performed by some of experimental processes such as focused ion beam (FIB) and scanning electron microscopy (SEM) [18]. These techniques were used to extract some of statistical functions like two-point correlation function. Results of reconstruction process were used to generate a representative volume element (RVE) model. This model would be used as an initial model for finite element analysis.

Previous studies have investigated effects of inclusions with ordinary shapes on mechanical and other physical properties of microstructures; however effects of different shapes of tessellations on mechanical behavior of a microstructure can be studied in a more distinct manner. In a step forward in this research, unlike previous studies, effects of particles with different geometrical shapes are investigated using finite element method. At the first step, a characteristic parameter for each shape is introduced as an initial approximation for shape effects. Afterwards, different steps of RVE preparation and generation of random structures are illuminated. Percolation analysis carried out by FEM sounds to result in more realistic results. The main objective of this work is to achieve procedure of changing the elastic properties of a nanocomposite using (a) honeycomb, (b) square, (c) triangular and (d) rhombus tessellations. The results derived by this analysis are then discussed and compared with theoretical limits in section 4 .

\section{Initial Approximation of the Inclusion Shape Effects}

In this section, a geometrical approximation about the effects of adding inclusions with different shapes on mechanical properties of the microstructure is investigated. As a rule of thumb, one can conclude that if the particles length in ' $y$ ' direction is longer than that in ' $x$ ' direction, adding them to the matrix have a greater effect in ' $y$ ' direction. To develop a simple geometrical approximation, a non-dimensional length can be defined as follows:

$$
\mathrm{D}_{\mathrm{c}}=\frac{\left.\mathrm{L}_{\mathrm{G}}\right|_{\mathrm{y}}}{\left.\mathrm{L}_{\mathrm{G}}\right|_{\mathrm{x}}}
$$

Where $D_{c}$ denotes the characteristic parameter associated with the effect of inclusion shape on mechanical properties of the microstructure stretched in ' $y$ ' direction. ' $\left.\mathrm{L}_{\mathrm{G}}\right|_{\mathrm{y}}$ ' is defined as the length of particle in ' $\mathrm{y}$ ' direction across the center of surface. Similarly, ' $\left.\mathrm{L}_{\mathrm{G}}\right|_{\mathrm{x}}$ ' is defined for the maximum length of particle in ' $\mathrm{x}$ ' direction. The characteristic parameter can be applied to have an estimation about the mechanical behavior of the microstructure in ' $x$ ' and ' $y$ ' direction when different shapes of inclusions are applied for reinforcement. Fig. 1 shows the parameters ' $\left.\mathrm{L}_{\mathrm{G}}\right|_{\mathrm{y}}$ ' and ' $\left.\mathrm{L}_{\mathrm{G}}\right|_{\mathrm{x}}$ ' for a triangular inclusion.

Fig. 1. Parameters ' $\left.\mathrm{L}_{\mathrm{G}}\right|_{\mathrm{y}}$ ' and ' $\left.\mathrm{L}_{\mathrm{G}}\right|_{\mathrm{x}}$ ' for a triangular inclusion.

Characteristic parameters for different inclusion shapes are listed in Table 1. It should be mentioned that inclusion with smaller characteristic length, show stiffer behavior in ' $x$ ' direction. 


\section{Finite Element Modeling of the Microstructure}

As a paradigmatic example, consider the case of a microstructure with different shapes of particles in it. In general, the microstructure function is somehow with perfect bonding between the matrix and particles. Effects of four different types of particle shapes on elastic properties of the microstructure, was investigated: Honeycomb, Rhombus, Square and Triangular. In each case, the matrix and particles was considered to have isotropic behavior. To specify different values for elastic modulus of the matrix and fiber, creating partitions with different mechanical properties can be helpful. Hence, in the next step, it is needed to create partitions on the microstructure based on the particle shapes. For square, triangular and rhombus shapes, the partitions were created using horizontal, vertical and angled lines on XY plane of the microstructure. For the honeycomb structure, at first coordinates of the center of particles were produced using a Python code. Then coordinates of these points were read in a MATLAB code and finally coordinates of the beginning and the end of the honeycomb particles edges were produced. Using these points, it is feasible to create honeycomb particles in microstructure. Fig. 2 shows two different partitioning states for the honeycomb and square particles.

Fig. 2. Two different partitioning states for the (a) Honeycomb, (b) Square particles.

The elastic modulus for inclusions is $\mathrm{E}_{\mathrm{i}}=1000 \mathrm{MPa}$, whereas the elastic modulus for matrix is $\mathrm{E}_{\mathrm{m}}=10 \mathrm{MPa}$. The Poisson's ratio for both inclusions and matrix is considered to be $v=0.3$. The upper and lower bound (limit) of elastic properties can be determined through the following equations respectively:

$$
\begin{gathered}
E_{L}=E_{i} V_{f}+E_{m}\left(1-V_{f}\right) \\
\frac{1}{E_{L}}=\frac{V_{f}}{E_{i}}+\frac{1-V_{f}}{E_{m}}
\end{gathered}
$$

\subsection{The Protocol for Generation of the Random Microstructure:}

A random number is assigned to each partition. If the random number is smaller than the volume fraction of the particles, the stiffer material will be assigned to the partition. Otherwise, the partition is considered to have the weaker elastic properties. At first, the microstructure includes 5\% volume fraction of the particles. In each step, volume fraction of particles in microstructure increases sequentially until it reaches to $95 \%$. The finite element process was performed for each step.

Elastic properties of the microstructure with different shapes of particles have been evaluated using three types of loading conditions: in-plane stretching in ' $x$ ' direction, in-plane stretching in ' $y$ ' direction and in-plane shear loading. In case of stretching in ' $x$ ' (' $y$ ') direction, a horizontal (vertical) displacement (equal to 0.001 strain) on the left (upper) edge of the microstructure was applied and on the right (lower) edge of the microstructure a boundary condition was applied to restraint any displacement in ' $x$ ' (' $y$ ') direction. In case of in-plane shear condition, the microstructure was subjected to an in-plane shear displacement (equal to 0.001 strain) on its left edge in the negative 
Fig. 3. Loading conditions on microstructure (a)- In-plane stretching condition, (b)- In-plane shear loading

ABAQUS commercial software (Abaqus/Standard; Simulia Dassault Systemes Simulia Corp. Providence, RI, USA) was used for finite element modeling and computations of the microstructure deformation under mentioned loading and boundary conditions. Three static steps for each type of loading were considered. Note that in this study, we calculate the stress and volume for each element in each step using finite element method. Following equations can be used as an alternative approach to calculate the elastic constants [8]:

$$
\begin{aligned}
& \mathrm{E}_{\mathrm{x}}=\mathrm{C}_{11}=\frac{\left(\sum_{\mathrm{i}=1}^{\mathrm{N}} \sigma_{11}^{\mathrm{i}} \mathrm{v}^{\mathrm{i}}\right)}{\varepsilon_{11}^{0} \mathrm{~V}} \\
& \mathrm{E}_{\mathrm{y}}=\mathrm{C}_{22}=\frac{\left(\sum_{\mathrm{i}=1}^{\mathrm{N}} \sigma_{22}^{\mathrm{i}} \mathrm{v}^{\mathrm{i}}\right)}{\varepsilon_{22}^{0} \mathrm{~V}} \\
& \mathrm{G}_{\mathrm{xy}}=\mathrm{C}_{12}=\frac{\left(\sum_{\mathrm{i}=1}^{\mathrm{N}} \sigma_{12}^{\mathrm{i}} \mathrm{v}^{\mathrm{i}}\right)}{\varepsilon_{12}^{0} \mathrm{~V}}
\end{aligned}
$$

Where $\mathrm{N}$ is the total number of elements, $\mathrm{v}^{\mathrm{i}}$ is the volume of $\mathrm{i}^{\text {th }}$ element, $\sigma^{\mathrm{i}}$ is the stress tensor associated with $\mathrm{i}^{\text {th }}$ element and $\varepsilon^{0}$ is the external strain tensor applied on the microstructure.

\section{Results and Discussion}

These models were analyzed based on the previous discussions and assumptions. The first goal is to find the trend of elastic modulus in stretching and shear mode for the microstructure. The comparison is performed for added particles with different geometry (shown in Figs. 4, 5 and 6). For the sake of completeness the elastic modulus curves are compared with the associated lower bound and upper bound limits. Fig. 4 shows the elastic modulus of microstructure in ' $x$ ' direction by considering the effect of different shapes for inclusions on the mechanical properties of the whole microstructure.

Fig. 4. Overall elastic modulus of the microstructure in ' $x$ ' direction. Comparison of the different effect of shapes of cells on the mechanical properties of the whole microstructure

It can be observed from Fig. 4 that the trend of elastic modulus in ' $x$ ' direction for all types of inclusion shapes is ascending by increase in volume fraction of particles. One can conclude that among the different shapes of inclusions, the Honeycomb shape has the best effect on enhancement of mechanical properties of the microstructure. After the honeycomb shape, the square, triangular and rhombus shapes can enhance the mechanical properties of the microstructure respectively. This is in a good level of consistency with the approximation pointed out in Sec. 2. 
Comparing different curves in Fig. 4, it is evident that in a microstructure with $30 \%$ volume fraction inclusions, the particles shape effects doesn't have a remarkable effect on stretching properties of the microstructure. It can be observed that by adding particles in ranges higher than $65 \%$ volume fractions, the elastic modulus in ' $x$ ' direction for all types of microstructures increases in a higher rate (Fig. 4). Also, Fig. 4 shows that for the ranges higher than $75 \%$ volume fraction, elastic modulus of the whole microstructure obviously converges to the upper bound. The dotted line in Fig. 4 shows the average of upper and lower bound limits. Taking into account the geometrical shape of particles, elastic moduli for all of the investigated structures reached to the average bound in ranges of volume fractions between $75 \%-85 \%$.

Fig. 5 shows the comparison between the elastic modulus of microstructure in ' $y$ ' direction with different particle shapes. As in the $E_{x}$, the elastic modulus in ' $y$ ' direction has an ascending trend for all types of inclusion shapes. Again, Fig. 5 shows a higher rate for the ascending trend of $\mathrm{E}_{\mathrm{y}}$ in ranges higher than $65 \%$ volume fraction and the modulus line is reached to the average of upper and lower bounds in ranges of $70 \%-80 \%$ volume fraction. Among the different shapes for the particles, the rhombus shape has a higher range for the elastic modulus in ' $y$ ' direction that is in a good agreement with the approximation relation pointed out in Sec. 2. It should be noted that this convergence resulted in a considerable percolation of the microstructures.

Fig.5. Overall elastic modulus of the microstructure in ' $y$ ' direction. Comparison of the different effect of shapes of cells on the mechanical properties of the whole microstructure

Fig. 6 shows the shear modulus of microstructure with different shapes of particles. In Fig. 6 (similar to Fig. 4), whenever the particles volume fraction is less than $30 \%$, the particles shape effects doesn't have a significant effect on shearing properties of the microstructure. Unlike the previous diagrams, the shear modulus diagram doesn't have an additive trend for each level of particles volume fraction. In Fig. 6, it is seen that the rhombus particles make the stiffest structure among other microstructures in shear loading condition. The effect of adding square particles on overall shearing behavior of microstructure is descending intensively when the volume fraction of particles is in a certain range $\left(0.7<\mathrm{V}_{\mathrm{f}}<0.8\right)$. For triangular particles the descending behavior of the shear modulus was evaluated in some of intermediate values of volume fraction $(0.45,0.65$ and 0.9$)$. The intensive descending behavior in shear modulus was not observed in case of using the rhombus particles. In this case, an intensive additive trend can be observed in $\mathrm{V}_{\mathrm{f}} \approx 65 \%$ instead. This effect can be observed in case of triangular particles with $\mathrm{V}_{\mathrm{f}} \approx 65 \%$.

Fig. 6. Overall shear modulus of the microstructure. Comparison of the different effect of shapes of cells on the mechanical properties of the whole microstructure

Fig. 7 shows the honeycomb microstructures for volume fractions from $5 \%$ to $95 \%$. It is obvious that for the ranges from $55 \%$ to $75 \%$ of volume fraction, noticeable clusters of the stiffer material are appeared and although one can conclude that the correlation of the stiffer material has improved the overall elastic properties. 
Fig.7. Honeycomb model overview (red cells are stiffer particles and green cells are matrix particles) for different volume fractions

Fig. 7 shows the honeycomb microstructures for volume fractions from $5 \%$ to $95 \%$. It is obvious that for the ranges from $55 \%$ to $75 \%$ of volume fraction, noticeable clusters of the stiffer material are appeared and although one can conclude that the correlation of the stiffer material has improved the overall elastic properties.

Fig. 8 shows the rhombus microstructures for volume fractions from $5 \%$ to $95 \%$. It should be mentioned that different shapes of particles in microstructures have different effect on percolation and consequently different elastic modulus values for each volume fraction is obtained. One can define a percolation threshold of elastic properties for each shape of particles but application of these thresholds can be discussable.

Fig. 8. Rhombus model overview (red cells are stiffer particles and green cells are matrix particles) for different volume fractions

Fig. 9, Fig. 10 and Fig. 11 show the stress contour plots in microstructure based on the shape of cells under mentioned loading and boundary conditions in volume fraction of $25 \%$ for the stiffer material.

Fig.9. Stress contour plots for microstructures with $25 \%$ volume fraction of the stiffer material. The left column shows $\mathrm{S}_{11}$, the middle column shows $S_{22}$ and the right column shows $S_{12}$. The first, second, third and fourth rows are associated with the honeycomb, rhombus, square and triangular microstructures respectively.

Fig.10. Stress contour plots for microstructures with $50 \%$ volume fraction of the stiffer material. The left column shows $\mathrm{S}_{11}$, the middle column shows $S_{22}$ and the right column shows $S_{12}$. The first, second, third and fourth rows are associated with the honeycomb, rhombus, square and triangular microstructures respectively.

Fig.11. Stress contour plots for microstructures with $75 \%$ volume fraction of the stiffer material. The left column shows $\mathrm{S}_{11}$, the middle column shows $S_{22}$ and the right column shows $S_{12}$. The first, second, third and fourth rows are associated with the honeycomb, rhombus, square and triangular microstructures respectively. 


\section{Conclusions}

In this paper effects of geometrical shape of inclusion on elastic properties of microstructures have been investigated. Finite element method is used to evaluate mechanical properties of the microstructure reinforced by inclusions with different shapes. FEM results show that based on shapes of inclusions, adding them to matrix enhances the mechanical properties of the whole microstructure in different states. The more particles added to the microstructure causes the more enhanced stretching properties in ' $x$ ' and ' $y$ ' directions, while this is not confirmed for shear modulus. In shear loading mode the microstructure is weakened in some ranges of volume fractions. In stretching mode in ' $x$ ' direction, the honeycomb particles have the most enhancing effect on mechanical properties of the microstructures. However, for stretching mode in ' $y$ ' direction the rhombus particles have the most enhancing effect on mechanical properties of microstructure. A comparison has also been made among the shear loading results. Unlike the stretching mode, the ascending trend for shear modulus has not been verified when the volume fraction of particles is increased. Rhombus particles had the maximum range of shear modulus among the other shapes. The ascending trend for high volume fractions also shows the effects of percolation of stiffer particles on the overall elastic properties of the microstructure. In the next steps, this research can be continued by investigating the effects of dissimilar particles in the overall behavior of microstructure.

\section{Acknowledgment}

The authors would like to acknowledge the funding for this project from the Iran National Science Foundation (INSF).

\section{References}

1. Qiao, R. and L.C. Brinson, Simulation of interphase percolation and gradients in polymer nanocomposites. Composites Science and Technology, 2009. 69(3): p. 491-499.

2. $\quad$ Kurahatti, R., et al., Defence applications of polymer nanocomposites. Defence Science Journal, 2010. 60(5): p. 551-563.

3. Wang, H., et al., Nanoreinforced polymer composites: 3D FEM modeling with effective interface concept. Composites Science and Technology, 2011. 71(7): p. 980-988.

4. Favier, V., H. Chanzy, and J. Cavaille, Polymer nanocomposites reinforced by cellulose whiskers. Macromolecules, 1995. 28(18): p. 6365-6367.

5. Celzard, A., et al., Critical concentration in percolating systems containing a high-aspect-ratio filler. Physical Review B, 1996. 53(10): p. 6209.

6. Niklaus, M. and H. Shea, Electrical conductivity and Young's modulus of flexible nanocomposites made by metalion implantation of polydimethylsiloxane: The relationship between nanostructure and macroscopic properties. Acta Materialia, 2011. 59(2): p. 830-840.

7. McLachlan, D.S., M. Blaszkiewicz, and R.E. Newnham, Electrical resistivity of composites. Journal of the American Ceramic Society, 1990. 73(8): p. 2187-2203.

8. Yves, R., et al., Applied RVE Reconstruction and Homogenization of Heterogeneous Materials2016: John Wiley \& Sons.

9. Ouali, N., J. Cavaillé, and J. Perez, Elastic, viscoelastic and plastic behavior of multiphase polymer blends. Plastics, Rubber and Composites Processing Applications, 1991: p. 55-60.

10. Seidel, G.D. and D.C. Lagoudas, A micromechanics model for the electrical conductivity of nanotube-polymer nanocomposites. Journal of Composite Materials, 2009. 43(9): p. 917-941.

11. Liu, H. and L.C. Brinson, Reinforcing efficiency of nanoparticles: a simple comparison for polymer nanocomposites. Composites Science and Technology, 2008. 68(6): p. 1502-1512.

12. Baxter, S.C., B.J. Burrows, and B.S. Fralick, Mechanical percolation in nanocomposites: Microstructure and micromechanics. Probabilistic Engineering Mechanics, 2015.

13. Asiaei, S., et al., Effects of carbon nanotubes geometrical distribution on electrical percolation of nanocomposites: a comprehensive approach. Journal of Reinforced Plastics and Composites, 2009. 
14. Baniassadi, M., et al., Incorporation of electron tunnelling phenomenon into 3D Monte Carlo simulation of electrical percolation in graphite nanoplatelet composites. Journal of Physics D: Applied Physics, 2011. 44(45): p. 455306.

15. Chen, X.-M., J.-W. Shen, and W.-Y. Huang, Novel electrically conductive polypropylene/graphite nanocomposites. Journal of materials science letters, 2002. 21(3): p. 213-214.

16. Weng, W.-G., et al., HDPE/expanded graphite electrically conducting composite. Composite Interfaces, 2004. 11(2): p. 131-143.

17. Baniassadi, M., et al., Using SAXS approach to estimate thermal conductivity of polystyrene/zirconia nanocomposite by exploiting strong contrast technique. Acta Materialia, 2011. 59(7): p. 2742-2748.

18. Sheidaei, A., et al., 3-D microstructure reconstruction of polymer nano-composite using FIB-SEM and statistical correlation function. Composites Science and Technology, 2013. 80: p. 47-54. 
Figure1. Parameters ' $\left.\mathrm{L}_{\mathrm{G}}\right|_{\mathrm{y}}$ ' and ' $\left.\mathrm{L}_{\mathrm{G}}\right|_{\mathrm{x}}$ ' for: a. Triangular, b. Square, c. Rhombus and d. Honeycomb inclusion 


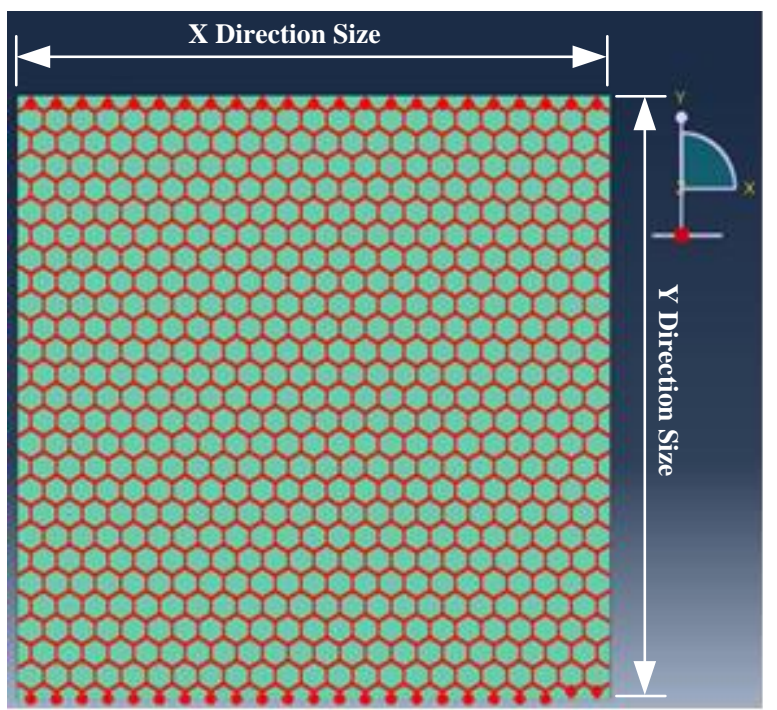

(a)

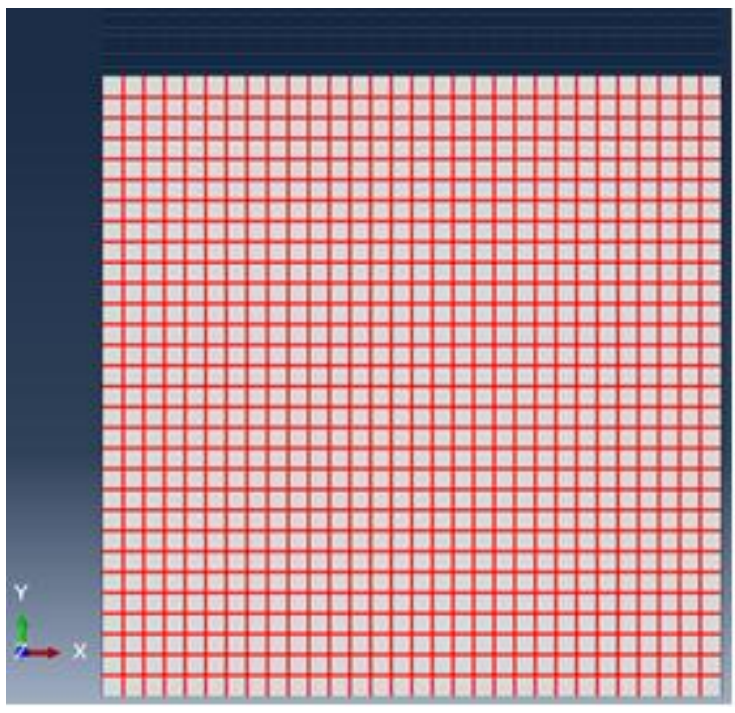

(b)

Fig. 2. Two different partitioning states for the (a) Honeycomb (Hexagonal Cells), (b) Square Cells. 


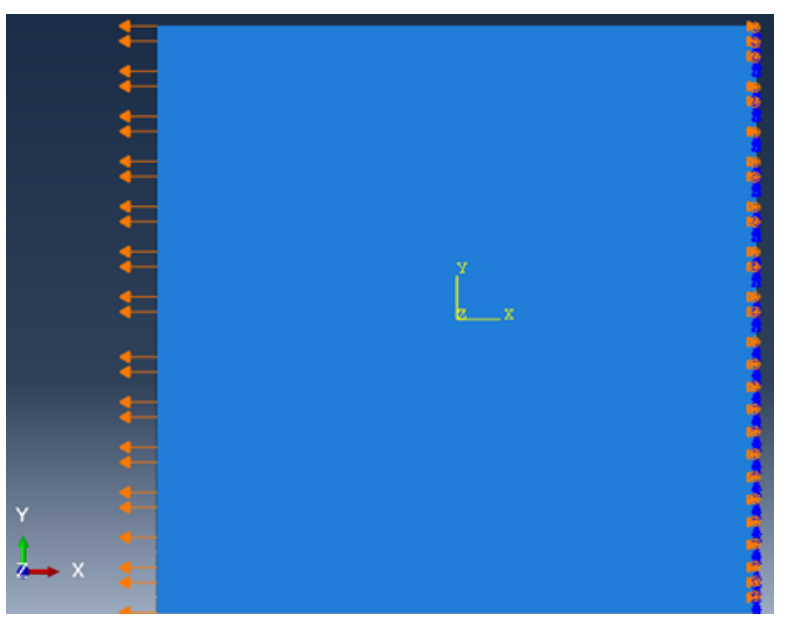

(a)

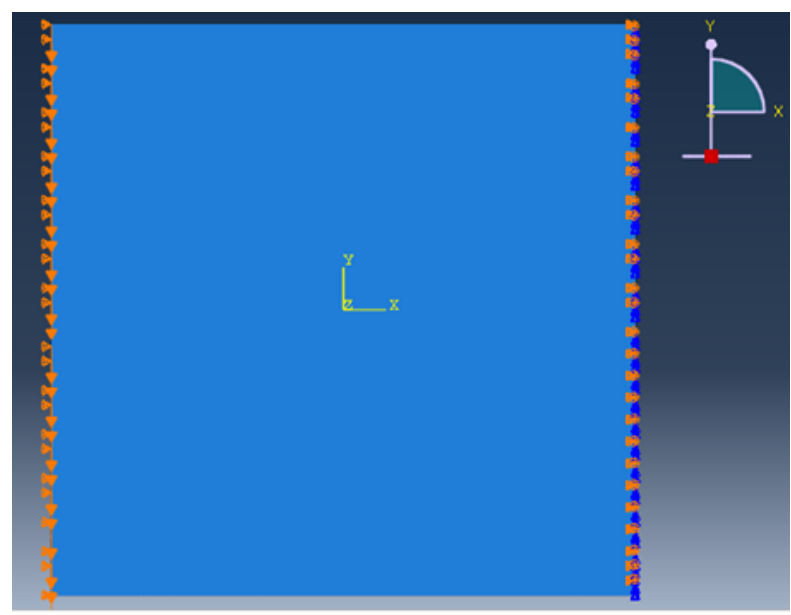

(b)

Fig. 3. Loading conditions on microstructure (a)- In-plane stretching condition, (b)- In-plane shear loading 


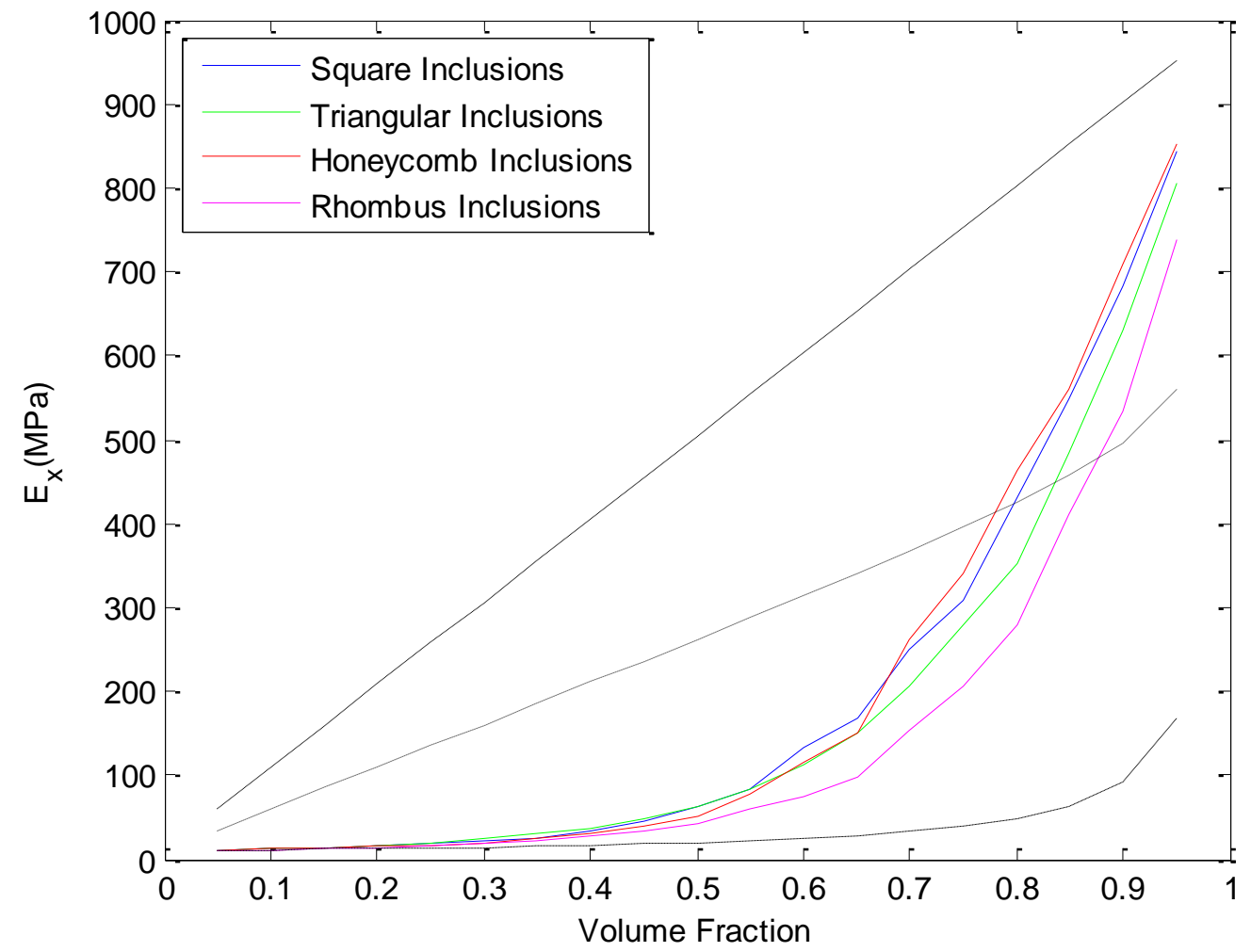

Figure. 4. Overall elastic modulus of the microstructure in ' $x$ ' direction. Comparison of the different effect of shapes of cells on the mechanical properties of the whole microstructure. Dashed lines from top to bottom represent the upper bound, upper \& lower bounds average and lower bound limits respectively. 


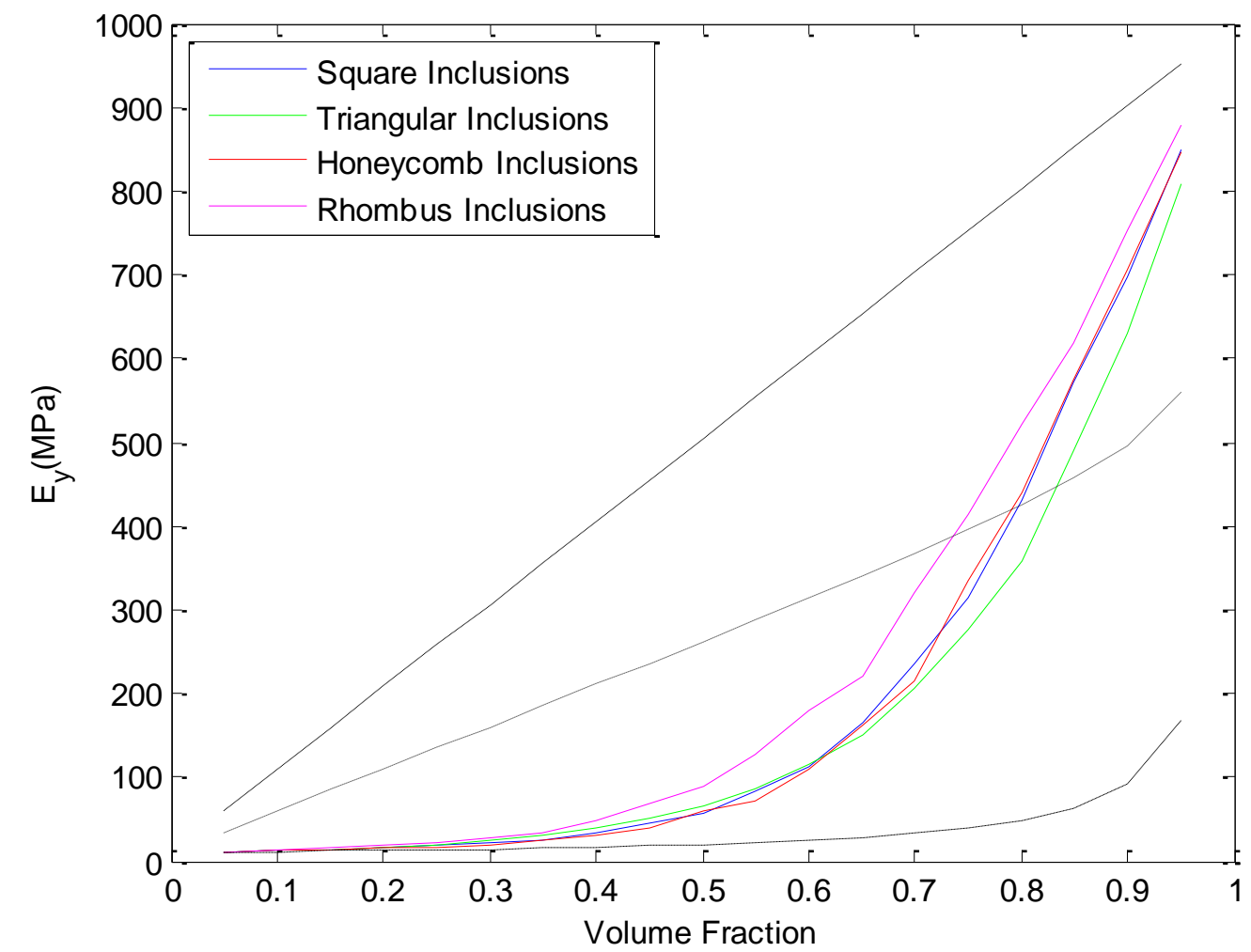

Fig. 5. Overall elastic modulus of the microstructure in ' $y$ ' direction. Comparison of the different effect of shapes of cells on the mechanical properties of the whole microstructure. Dashed lines from top to bottom represent the upper bound, upper \& lower bounds average and lower bound limits respectively. 


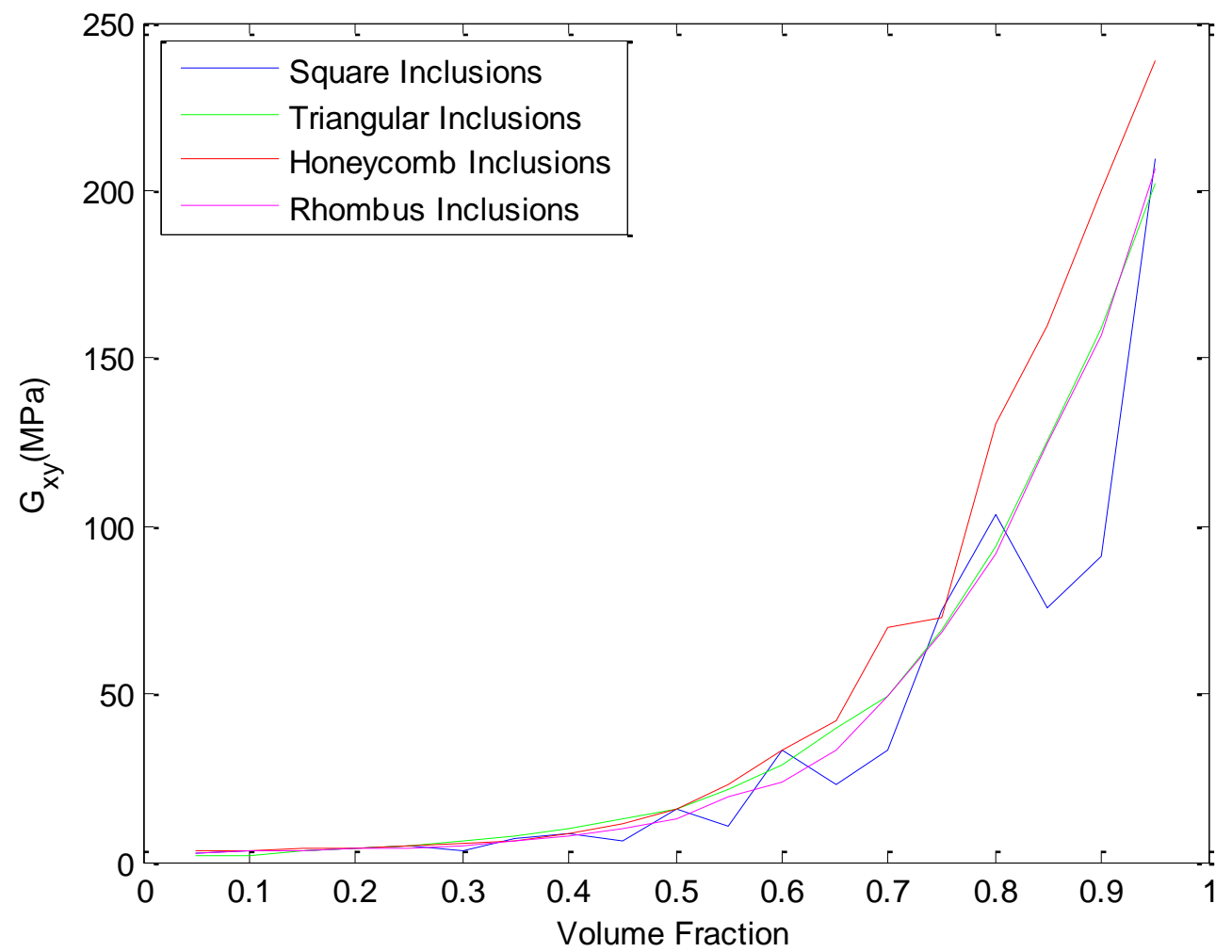

Fig. 6. Overall shear modulus of the microstructure. Comparison of the different effect of shapes of cells on the mechanical properties of the whole microstructure 


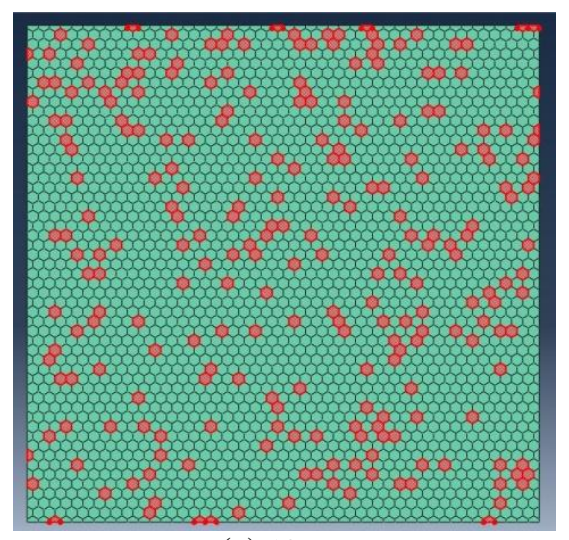

(a) $10 \%$

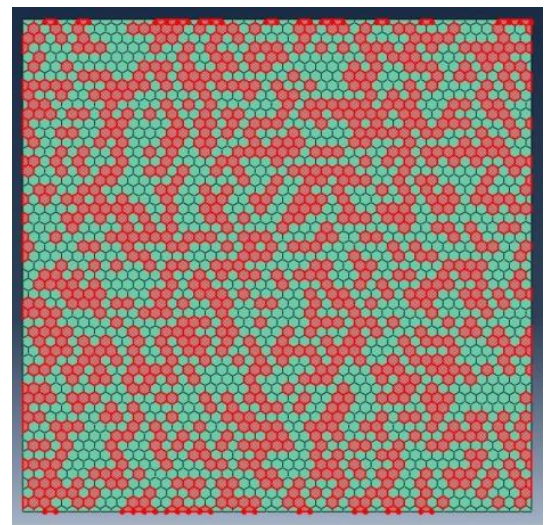

(d) $40 \%$

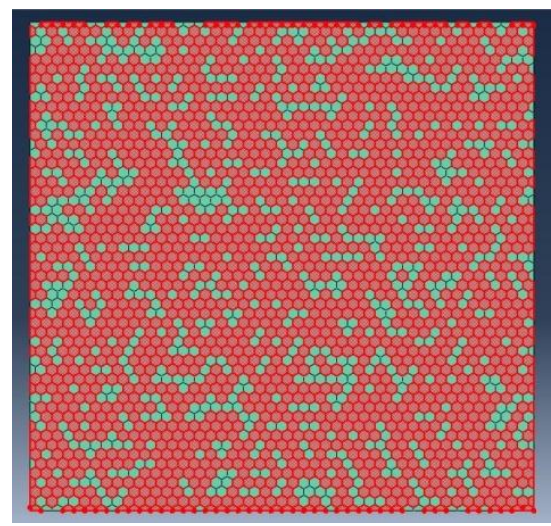

(g) $70 \%$

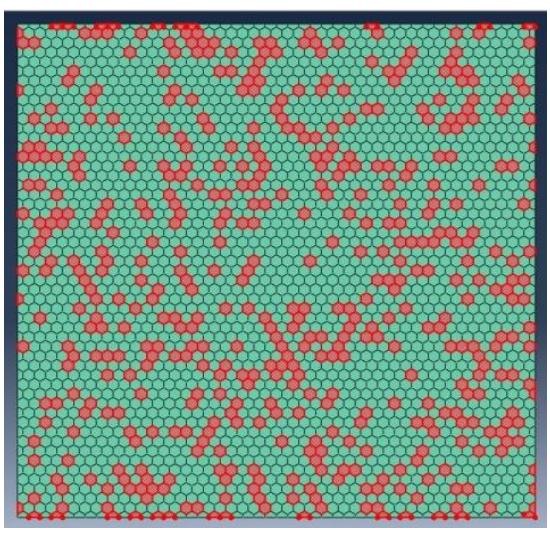

(b) $20 \%$

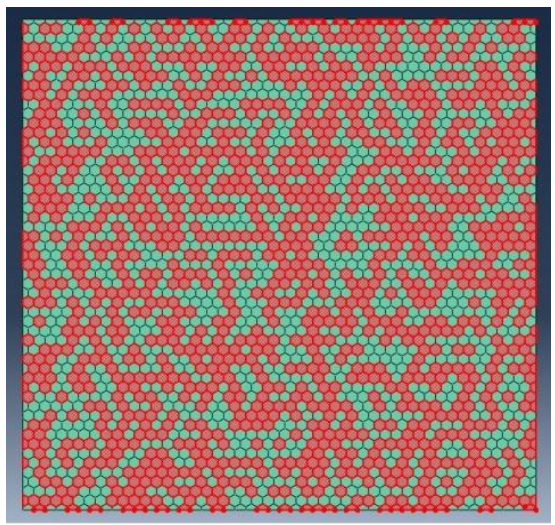

(e) $50 \%$

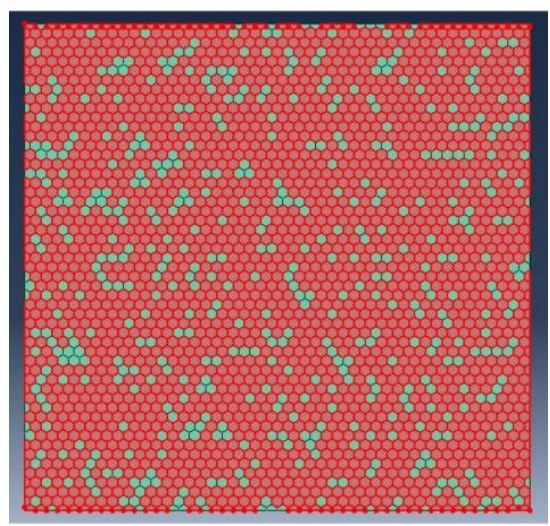

(h) $80 \%$

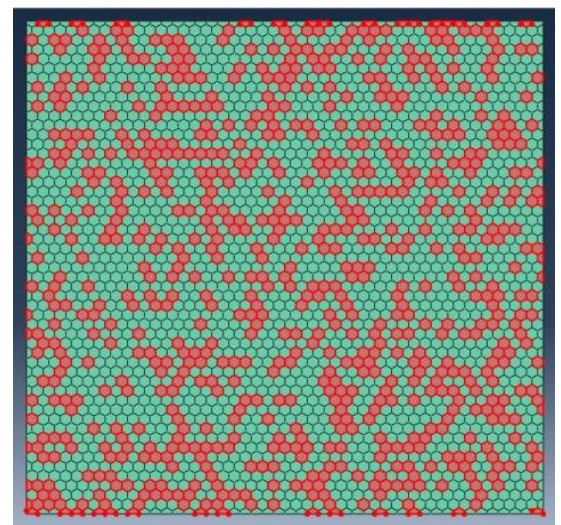

(c) $30 \%$

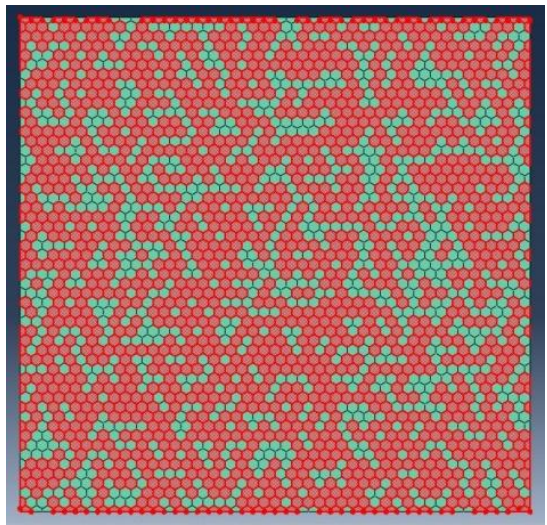

(f) $60 \%$

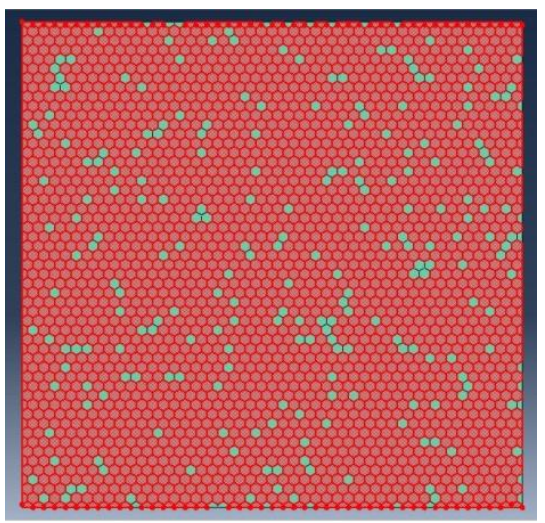

(i) $90 \%$

Fig.7. Honeycomb model overview (red cells are stiffer particles and green cells are matrix particles) for different volume fractions 


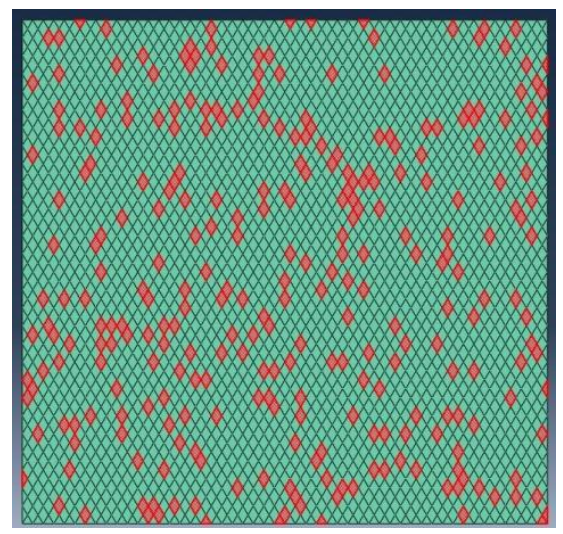

(b) $10 \%$

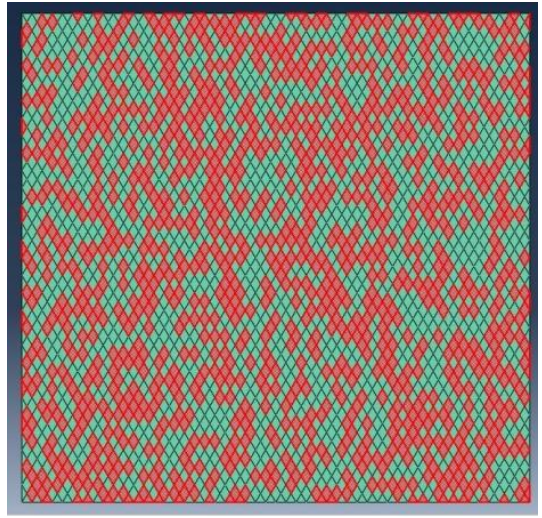

(h) $40 \%$

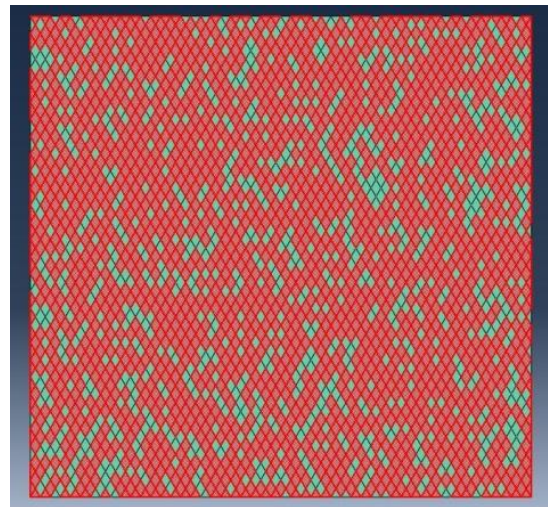

(n) $70 \%$

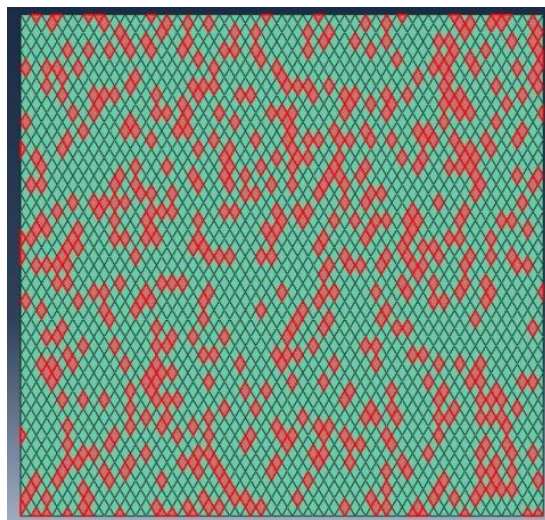

(d) $20 \%$

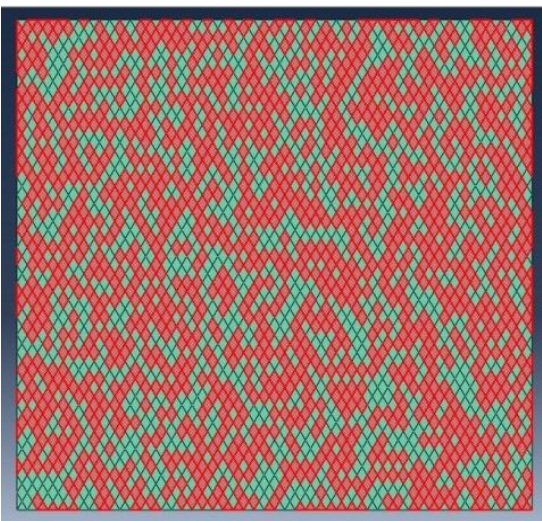

(j) $50 \%$

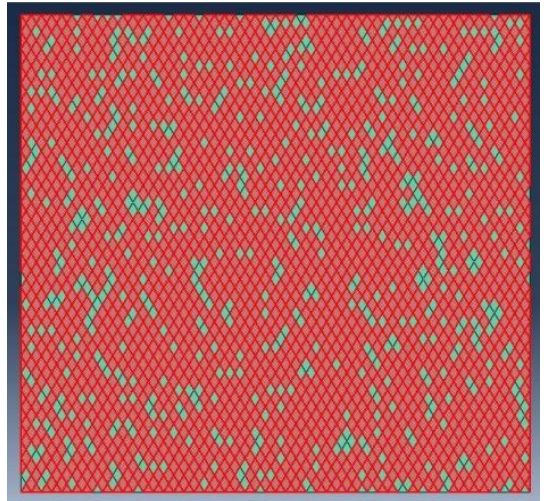

(p) $80 \%$

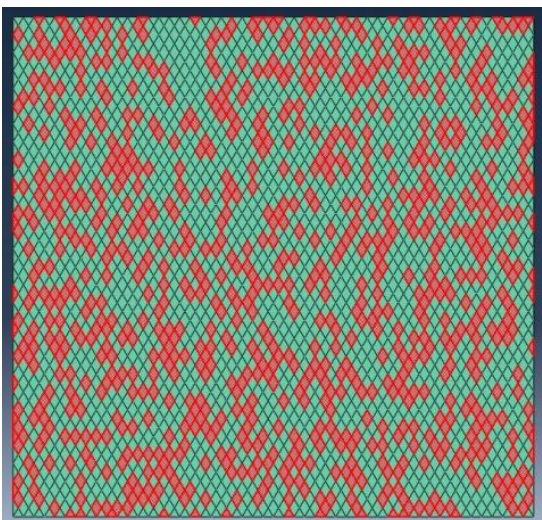

(f) $30 \%$

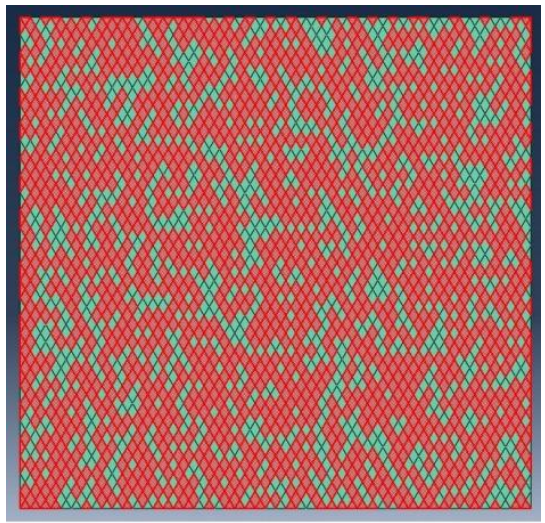

(l) $60 \%$

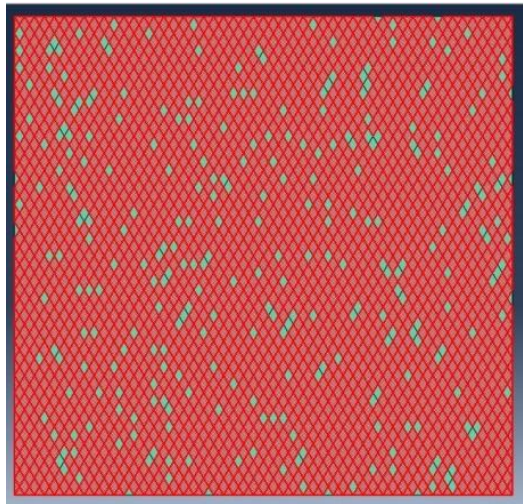

(r) $90 \%$

Fig. 8. Rhombus model overview (red cells are stiffer particles and green cells are matrix particles) for different volume fractions 

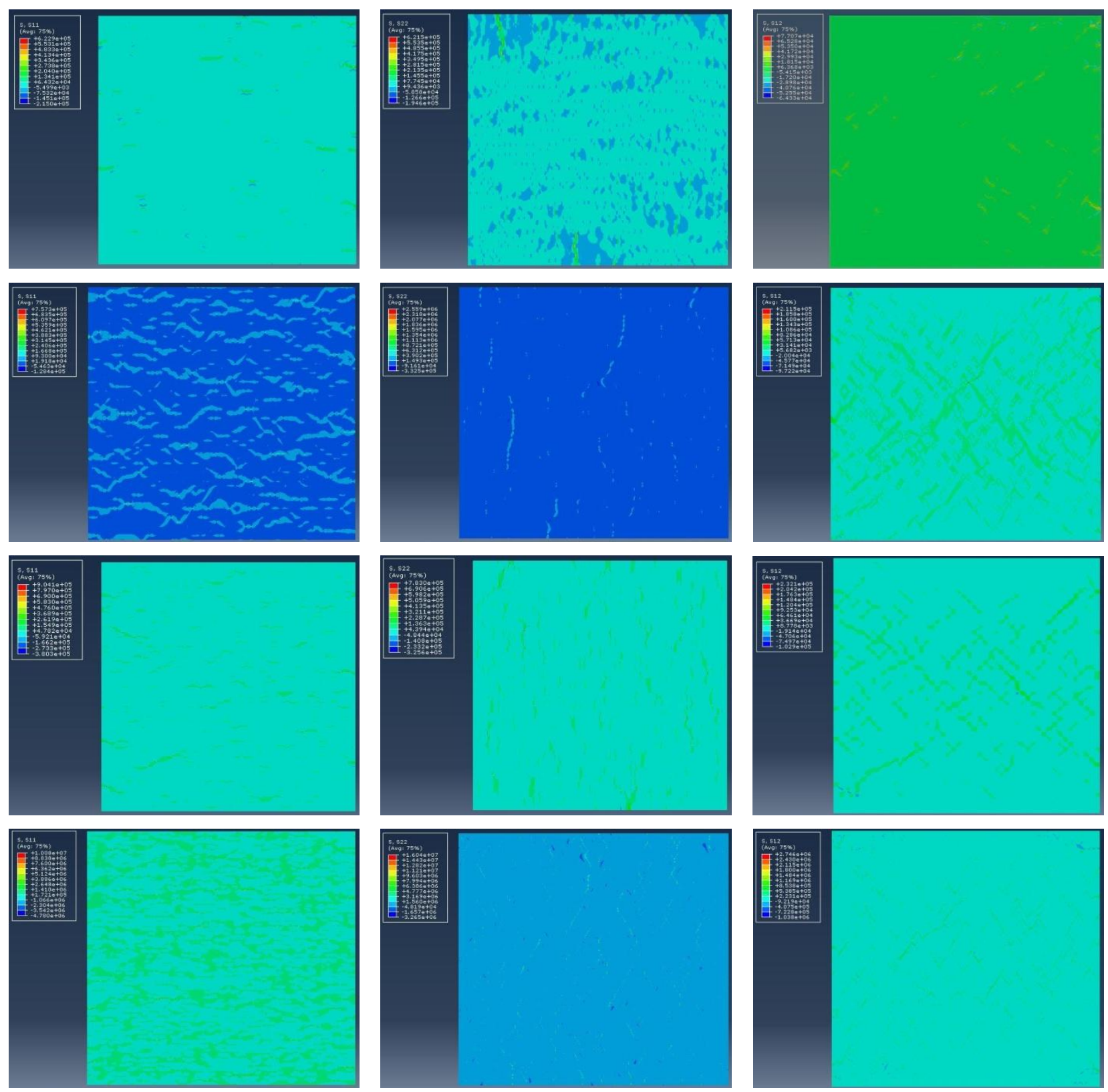

Fig.9. Stress contour plots for microstructures with $25 \%$ volume fraction of the stiffer material. The left column shows $\mathrm{S}_{11}$, the middle column shows $S_{22}$ and the right column shows $S_{12}$. The first, second, third and fourth rows are associated with the honeycomb, rhombus, square and triangular microstructures respectively. 

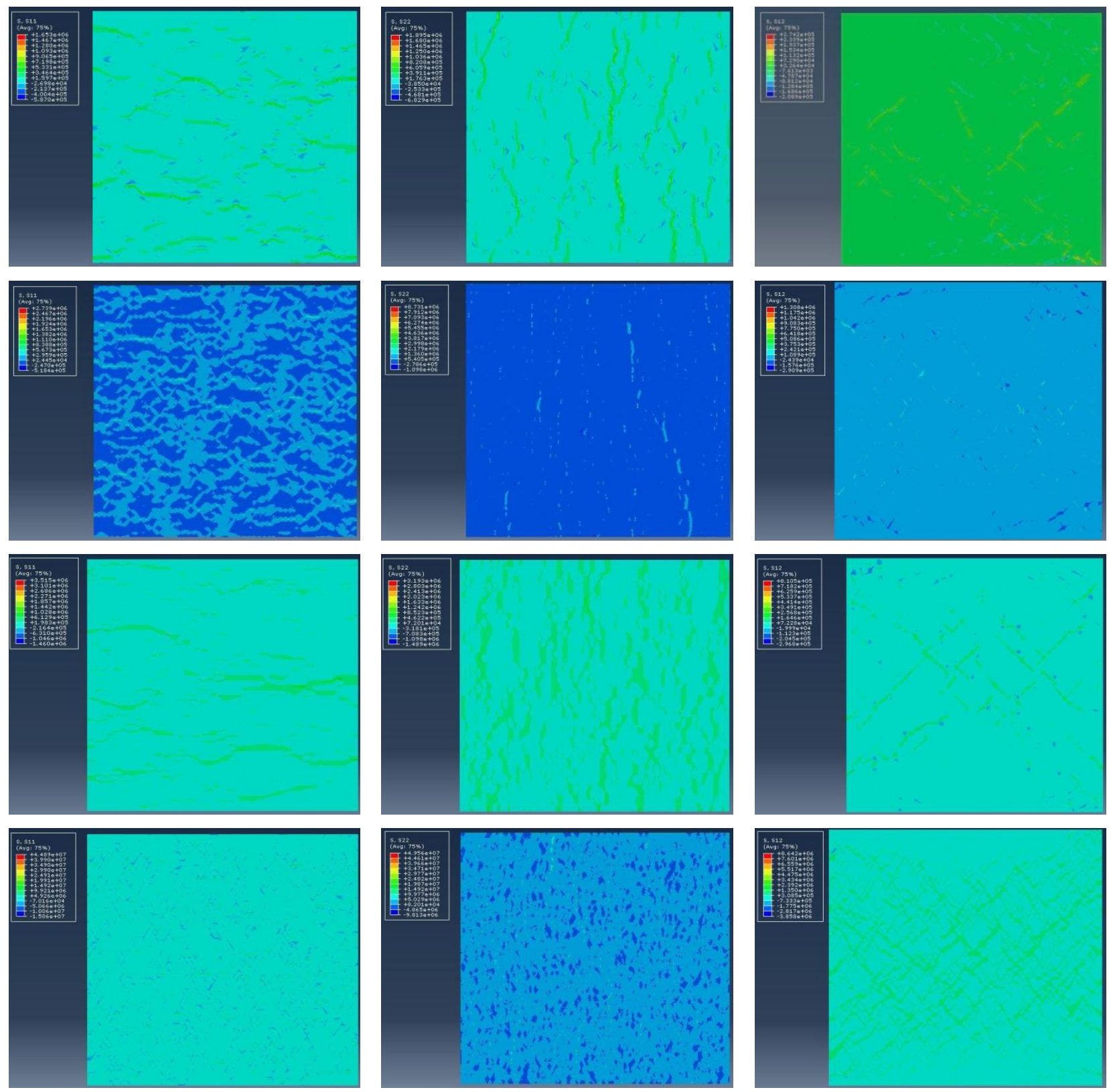

Fig.10. Stress contour plots for microstructures with 50\% volume fraction of the stiffer material. The left column shows $S_{11}$, the middle column shows $S_{22}$ and the right column shows $S_{12}$. The first, second, third and fourth rows are associated with the honeycomb, rhombus, square and triangular microstructures respectively 

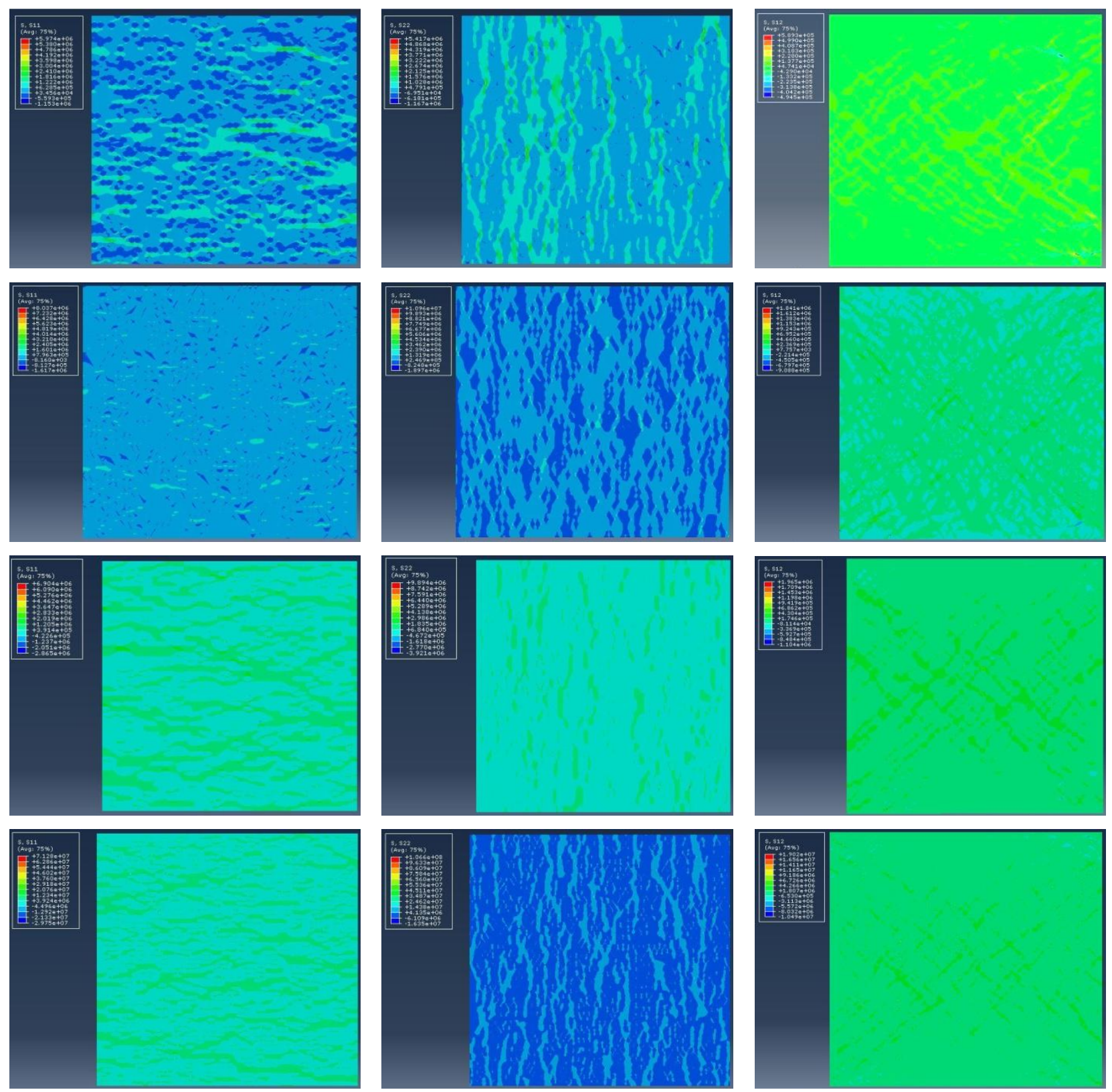

Fig.11. Stress contour plots for microstructures with $75 \%$ volume fraction of the stiffer material. The left column shows $\mathrm{S}_{11}$, honeycomb, rhombus, square and triangular microstructures respectively. 


\begin{tabular}{|c|c|c|c|c|}
\hline $\begin{array}{c}\text { Particle } \\
\text { Type }\end{array}$ & Honeycomb & \multicolumn{2}{c|}{ Square } & Triangle \\
\hline $\begin{array}{c}\text { Particle } \\
\text { Shape }\end{array}$
\end{tabular}


Table 2. Modeling characteristics for each type of microstructure

\begin{tabular}{ccccc}
\hline & Square & Honeycomb & Triangular & Rhombus \\
\hline $\begin{array}{c}\text { X direction size } \\
\text { (Unit of length) }\end{array}$ & 50 & 79.6743371482 & 50 & 50 \\
\hline $\begin{array}{c}\text { Y direction size } \\
\text { (Unit of length) }\end{array}$ & 50 & 78 & 48.497422612 & 48.497422612 \\
\hline Number of meshes & 422500 & 1216891 & 514201 & 421552 \\
\hline Cells number & 2500 & 2464 & 5656 & 2878 \\
\hline
\end{tabular}

344 